\title{
Exohydrogenated single-wall carbon nanotubes
}

\author{
T. Yildirim, ${ }^{1}$ O. Gülseren, ${ }^{1,2}$ and S. Ciraci ${ }^{3}$ \\ ${ }^{1}$ NIST Center for Neutron Research, National Institute of Standards and Technology, Gaithersburg, Maryland 20899 \\ ${ }^{2}$ Department of Materials Science and Engineering, University of Pennsylvania, Philadelphia, Pennsylvania 19104 \\ ${ }^{3}$ Physics Department, Bilkent University, Ankara, Turkey
}

(Received 8 February 2001; revised manuscript received 10 May 2001; published 17 July 2001)

\begin{abstract}
An extensive first-principles study of fully exohydrogenated zigzag $(n, 0)$ and armchair $(n, n)$ single-wall carbon nanotubes $\left(\mathrm{C}_{n} \mathrm{H}_{n}\right)$, polyhedral molecules including cubane, dodecahedrane, and $\mathrm{C}_{60} \mathrm{H}_{60}$ points to crucial differences in the electronic and atomic structures relevant to hydrogen storage and device applications. $\mathrm{C}_{n} \mathrm{H}_{n}$ 's are estimated to be stable up to the radius of a $(8,8)$ nanotube, with binding energies proportional to $1 / R$. Attaching a single hydrogen to any nanotube is always exothermic. Hydrogenation of zigzag nanotubes is found to be more likely than armchair nanotubes with similar radius. Our findings may have important implications for selective functionalization and finding a way of separating similar radius nanotubes from each other.
\end{abstract}

DOI: 10.1103/PhysRevB.64.075404

PACS number(s): 61.48.+c, 61.46.+w, 61.50.Ah, 71.15.-m

Carbon nanotubes ${ }^{1}$ exhibit very unusual structural and electronic properties, suggesting a wide variety of technological applications, ${ }^{2,3}$ including the storage of hydrogen where the large effective surface area promises a large absorption capacity. ${ }^{4-10}$ Unfortunately, the studies to date report conflicting results. While some laboratories ${ }^{4,5}$ report hydrogen storage densities up to $10 \mathrm{wt} \% \%$ other laboratories report $^{6,7}$ only 0.4 wt. \% on the same system. Theories based on physisorption have failed to predict such high uptake. ${ }^{11}$ To the best of our knowledge, studies of hydrogen chemisorption in nanotubes are very limited ${ }^{12,13}$ and are clearly needed to have a better understanding of hydrogen and nanotube system.

Hydrogen-carbon interactions have been studied extensively both theoretically and experimentally for many interesting polyhedral molecules, such as cubane $\left(\mathrm{C}_{8} \mathrm{H}_{8}\right),{ }^{14-16}$ dodecahedrane $\left(\mathrm{C}_{20} \mathrm{H}_{20}\right),{ }^{17}$ and various isomers ${ }^{18,19}$ of $\mathrm{C}_{60} \mathrm{H}_{n}$ (see Fig. 1). Despite its very strained $90^{\circ} \mathrm{CCC}$-bond angle cubane has been synthesized successfully ${ }^{14}$ [Fig. 1(a)]. Similarly, dodecahedrane and various isomers of $\mathrm{C}_{60} \mathrm{H}_{n}$ (up to $n$ $=32$ ) have been also synthesized. ${ }^{18}$ These novel polyhedral molecules that represent the zero-dimensional case exhibit many interesting properties. However, due to the onedimensional nature and the curvature of carbon nanotubes, the hydrogen-carbon interactions in these systems may be quite different than those in polyhedral molecules. Therefore, it is important to know if it is also possible to hydrogenate carbon nanotubes in a similar way and if so what their structural and electronic properties would be. This paper addresses this important issue by performing extensive firstprinciples calculations and shows that the chemisorption of hydrogen is dependent on the radius and chirality of the nanotubes. Theoretical predictions from first-principles studies played an important role in guiding experimental studies in the past ${ }^{16,20}$ and we expect that many findings reported here may have important implications in this interesting system as well.

In order to obtain a reasonably complete understanding, we studied a very large number of systems including zigzag $(n, 0)(n=7,8,9,10$, and 12) and armchair $(m, m)(m=4$,
$5,6,8$, and 10) nanotubes ${ }^{21}$ as well as cubane, dodecahedrane, $\mathrm{C}_{60} \mathrm{H}_{60}$, and finally hydrogenated graphene sheet (i.e. an infinite limit of the tube radius). The first-principles total energy and electronic structure calculations were carried out using the pseudopotential plane wave method. ${ }^{22}$ The results have been obtained within the generalized gradient approximation $^{23}$ (GGA). This method has already been applied to many carbon systems, including fullerenes and cubane with remarkable success. ${ }^{16,20}$ We used plane waves with an energy cutoff of $500 \mathrm{eV}$. With this cutoff and using ultrasoft pseudopotentials for carbon, ${ }^{24}$ the total energy converges within $0.5 \mathrm{meV} /$ atom. Interactions between molecules or nanotubes in periodic cells are avoided by using large supercells. The supercell parameters are chosen such that the closest $\mathrm{H}$-H distance is $6 \AA$. For molecular calculations the Brillouin zone integration is carried out at the $\Gamma$ point. For nanotubes, we used $k$-point spacing of $d k \approx 0.02 \AA^{-1}$, gen-
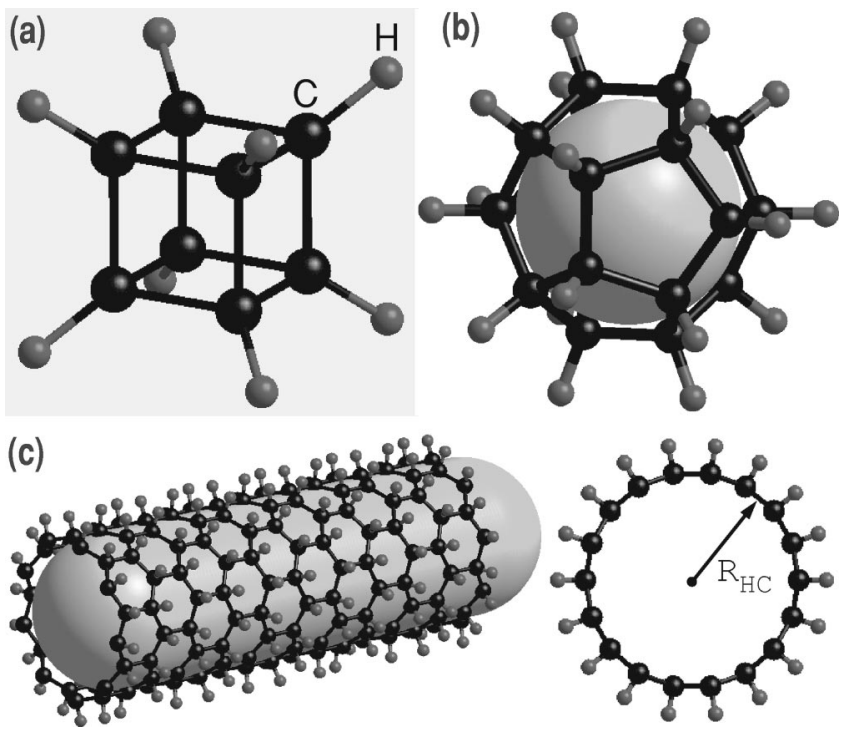

FIG. 1. Three different polyhedra of carbon and hydrogen; (a) cubane $\left(O_{h}\right)$, (b) dodecahedrane $\left(I_{h}\right)$, and (c) a side and top view of a single-wall exohydrogenated carbon nanotube. 
erating 5 and 10 special $k$ points along the tube axis for zigzag and armchair nanotubes, respectively. ${ }^{25}$ All carbon and hydrogen positions were relaxed without assuming any symmetry. For nanotube calculations, the $c$ axis of the supercell (corresponding to the tube axis) is also optimized.

In principle, there are an infinite number of isomers depending on the locations of hydrogen atoms (i.e., endohydrogenation if they are inside the tube and exohydrogenation if they are outside) as well as the amount of hydrogen coverage. Endohydrogenation, alternating endohydrogenationexohydrogenation, and various half coverage cases are being studied and the results will be published elsewhere. ${ }^{26}$ Here, we consider the case of full coverage where all carbon atoms in a nanotube are hybridized with hydrogen atoms from outside of the tube as shown in Fig. 1(c). We refer to this isomer as a fully exohydrogenated carbon nanotube.

First, the equilibrium orientations of the $\mathrm{CH}$ bonds were determined starting with all the $\mathrm{CH}$ bonds radially outward [Fig. 1(c)]. Using this configuration, we studied a single $\mathrm{CH}-$ bond orientational dependence of the potential energy surface. Figure 2 shows the calculated energy curves as a single $\mathrm{CH}$ bond is rotated along two high-symmetry directions for both zigzag and armchair nanotubes. For armchair $(n, n)$ nanotubes, the optimum orientation is obtained when the $\mathrm{CH}$ bond is tilted about the tube axis (i.e., $c$ axis). Hence in the fully optimized structure, $\mathrm{CH}$ bonds tilt in opposite directions around the $c$ axis alternatively. For the zigzag nanotubes, the optimum orientation is obtained when the $\mathrm{CH}$ bond is tilted towards the $c$ axis. Therefore, the lowest energy configuration for zigzag tubes has $\mathrm{CH}$ bonds tilted towards plus and minus $c$ axis alternatively. Having located the $\mathrm{CH}$-bond orientations in this way, we next let all the carbon and hydrogen atoms along the $c$ axis vary to obtain the final optimum structures.

Table I summarizes the parameters obtained for fully optimized structures. Upon hybridization of carbons with hydrogens, the CC-bond length $\left(d_{\mathrm{CC}}\right)$ increases from $\approx 1.4 \AA$ to $\approx 1.55 \AA$. The latter is typical for $s p^{3} \mathrm{CC}$ bonds. The increase in $d_{\mathrm{CC}}$ results in an increase in the tube radius $\left(R_{\mathrm{HC}}\right)$ by about $13-16 \%$ for armchair nanotubes and by about $15-$ $17 \%$ for zigzag nanotubes. Interestingly, these values are almost twice of those found for the polyhedral molecules. Moreover, the value of $d_{\mathrm{CC}}$ increases slightly (by about $0.03 \AA$ ) with increasing tube radius. The $\mathrm{CH}$-bond length $\left(d_{\mathrm{CH}} \approx 1.09 \AA\right)$ is also found to have weak dependence on the tube radius. Using projection techniques we estimated the total charge transfer from hydrogen to carbon to be around 0.26 electrons for nanotubes and 0.3 electrons for polyhedral molecules.

The most important difference between zigzag and armchair nanotubes is found in the local CCH-bond angles $\left(\alpha_{\mathrm{CCH}}\right)$. Even though one of these angles is about the same for both types of nanotubes, the second angle in zigzag nanotubes is always larger than that in armchair nanotubes. This implies that the CCH-bond angles are more frustrated in armchair nanotubes than in zigzag nanotubes and therefore deviate more from the ideal tetrahedral $s p^{3}$ bond angle of $109.5^{\circ}$. This observation suggests that hydrogenated armchair nanotubes will have higher energy and therefore they
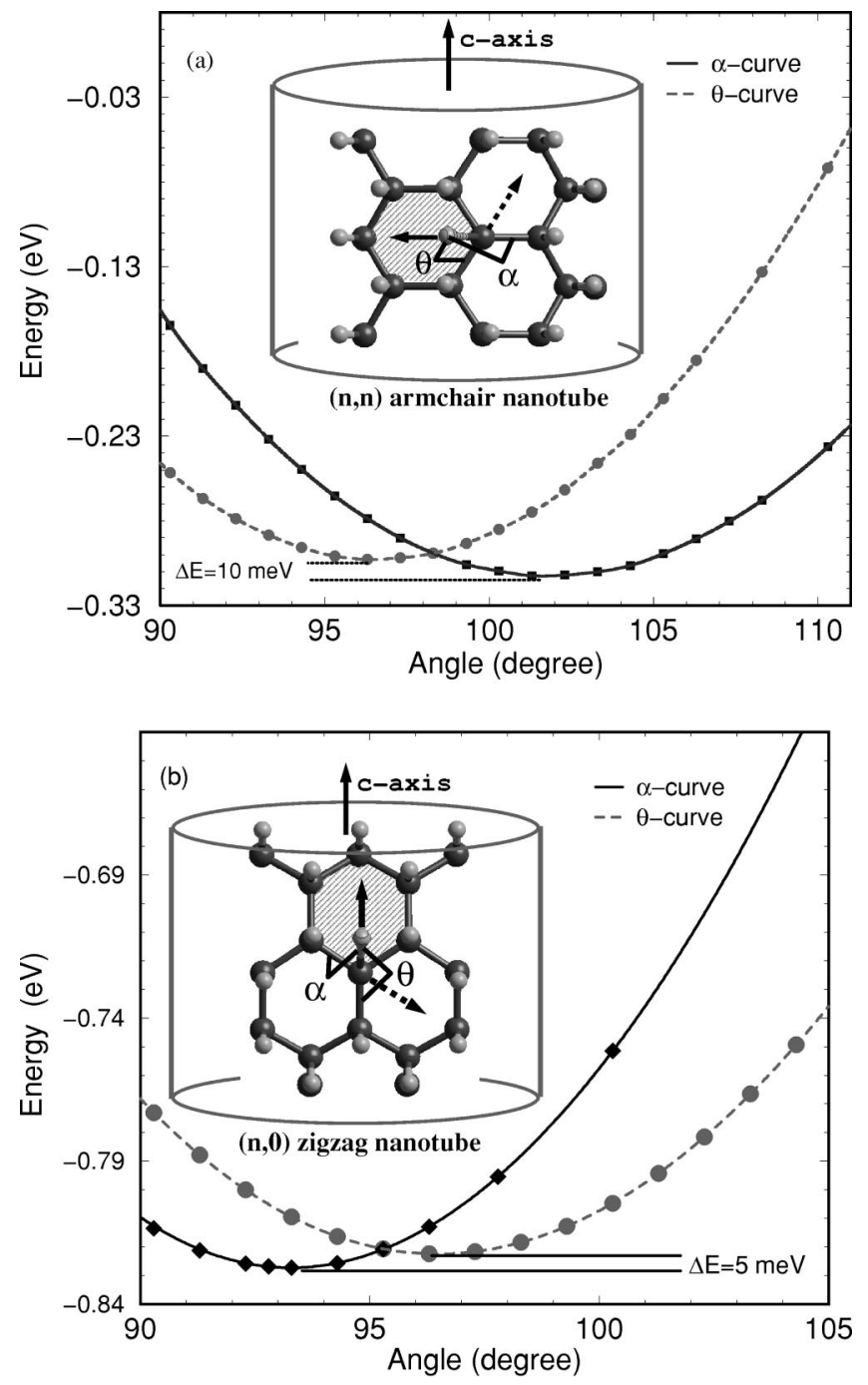

FIG. 2. Energy curves as a $\mathrm{CH}$ bond is rotated towards the indicated arrows for armchair (top) and zigzag nanotubes (bottom) starting from $\theta=\alpha$. The minimum energy is found when the $\mathrm{CH}$ bond is tilted toward the shaded hexagons. The zero of energy was taken to be arbitrary.

are less stable than zigzag nanotubes. Unlike $\mathrm{CCH}$-bond angles, CCC-bond angles have a weak radius dependence and are about the same for both types of nanotubes.

The stability and energetics of CH-bond formation are derived from the average binding energy per atom for exohydrogenated nanotubes defined as

$$
E_{B}=\left(E_{\mathrm{C}_{n} \mathrm{H}_{n}}-E_{\mathrm{C}}-n E_{\mathrm{H}}\right) / n .
$$

Here $E_{\mathrm{C}_{n} \mathrm{H}_{n}}, E_{\mathrm{C}}$, and $E_{\mathrm{H}}$ are the total energies of the fully optimized exohydrogenated nanotube, nanotube alone, and hydrogen atom, respectively. According to this definition, a stable system will have a negative binding energy. Figure 3 (a) shows the radius dependence of $E_{B}$ for nanotubes and polyhedral molecules (see inset). Two interesting observations are apparent. First, as shown by solid and dotted lines, the binding energies can be very well described by a oneparameter fit: 
TABLE I. Various parameters of the fully optimized structures of exohydrogenated armchair and zigzag carbon nanotubes and other polyhedral molecules. For graphene (i.e., $\left.R_{\mathrm{HC}} \rightarrow \infty\right) d_{\mathrm{CH}}$ and $d_{\mathrm{CC}}$ are $1.066 \AA$ and $1.622 \AA$, respectively.

\begin{tabular}{lcccccc}
\hline \hline Material & Formula & $R_{\mathrm{HC}}\left(R_{\mathrm{C}}\right)(\AA)$ & $d_{\mathrm{CH}}(\AA)$ & $d_{\mathrm{CC}}(\AA)$ & $\alpha_{\mathrm{CCH}}(\operatorname{deg})$ & $\alpha_{\mathrm{CCC}}(\operatorname{deg})$ \\
\hline$(4,4)$ & $\mathrm{C}_{16} \mathrm{H}_{16}$ & $3.103(2.734)$ & 1.090 & $1.541,1.567$ & $96.70,98.60$ & $112.77,120.69$ \\
$(5,5)$ & $\mathrm{C}_{20} \mathrm{H}_{20}$ & $3.885(3.394)$ & 1.087 & $1.549,1.575$ & $94.82,97.15$ & $113.18,121.50$ \\
$(6,6)$ & $\mathrm{C}_{24} \mathrm{H}_{24}$ & $4.698(4.061)$ & 1.084 & $1.557,1.594$ & $93.35,96.30$ & $113.30,122.00$ \\
$(8,8)$ & $\mathrm{C}_{32} \mathrm{H}_{32}$ & $6.228(5.400)$ & 1.079 & $1.567,1.594$ & $92.16,94.85$ & $114.62,121.95$ \\
$(10,10)$ & $\mathrm{C}_{40} \mathrm{H}_{40}$ & $7.780(6.755)$ & 1.077 & $1.574,1.600$ & $91.40,94.00$ & $115.40,121.76$ \\
$(7,0)$ & $\mathrm{C}_{28} \mathrm{H}_{28}$ & $3.180(2.765)$ & 1.092 & $1.549,1.553$ & $96.40,102.25$ & $113.95,125.90$ \\
$(8,0)$ & $\mathrm{C}_{32} \mathrm{H}_{32}$ & $3.641(3.146)$ & 1.090 & $1.553,1.557$ & $95.22,101.60$ & $114.12,127.00$ \\
$(9,0)$ & $\mathrm{C}_{16} \mathrm{H}_{16}$ & $4.111(3.557)$ & 1.089 & $1.553,1.566$ & $94.32,101.14$ & $114.27,127.58$ \\
$(10,0)$ & $\mathrm{C}_{40} \mathrm{H}_{40}$ & $4.571(3.912)$ & 1.087 & $1.556,1.572$ & $93.60,100.54$ & $114.48,127.85$ \\
$(12,0)$ & $\mathrm{C}_{48} \mathrm{H}_{48}$ & $5.467(4.695)$ & 1.084 & $1.557,1.576$ & $92.66,99.340$ & $115.11,127.67$ \\
Cubane & $\mathrm{C}_{8} \mathrm{H}_{8}$ & $1.345(1.267)$ & 1.087 & 1.553 & 125.26 & 90.0 \\
Dodecahedrane & $\mathrm{C}_{20} \mathrm{H}_{20}$ & $2.157(2.0)$ & 1.090 & 1.539 & 110.9 & 108.0 \\
Fullerene & $\mathrm{C}_{60} \mathrm{H}_{60}$ & $3.827(3.510)$ & 1.088 & $1.536,1.550$ & $101.1,101.9$ & $108.1,120.0$ \\
\hline \hline
\end{tabular}

$$
E_{B}=E_{0}-C(n, m) / R_{\mathrm{HC}},
$$

where $E_{0}$ is the limit $R_{\mathrm{HC}} \rightarrow \infty$ (i.e., graphene) and calculated to be $-1.727 \mathrm{eV}$. The fit results for $C(n, m)$ are given in Fig. 3(a) for zigzag and armchair nanotubes. The inset to Fig. 3(a) shows that while $E_{B}$ for cubane falls on the same curve as nanotubes, dodecahedrane and $\mathrm{C}_{60} \mathrm{H}_{60}$ have lower energies than nanotubes due to the their more spherical shape.

The second interesting observation in Fig. 3(a) is that the binding energies of zigzag nanotubes are always lower than those in armchair nanotubes with a similar radius by about $30 \mathrm{meV} /$ atom. As discussed above, this is a natural result of the fact that the $\mathrm{CCH}$-bond angles in zigzag nanotubes are closer to the optimum tetrahedral $s p^{3}$ bonding than those in armchair nanotubes. We expect this observation is also valid for hybridization of nanotubes with other elements, such as $\mathrm{Cl}$ and $\mathrm{F}$, and this may have important implications for separating similar radius nanotubes from each other by selective chemical functionalization.

Even though $\mathrm{C}_{n} \mathrm{H}_{n}$ nanotubes are found to be stable with respect to a pure carbon nanotube $\left(\mathrm{C}_{n}\right)$ and $n H$ atoms for all values of the radius, it is of interest to see if they are also stable against breaking a single $\mathrm{CH}$ bond. We, therefore, calculated energies of fully optimized hydrogenated nanotubes after breaking one of the $\mathrm{CH}$ bonds and putting the $\mathrm{H}$ atom at the center of supercell as shown in the top inset to Fig. 3(b). Calculated values of the energy differences $\Delta E_{B}$, for zigzag nanotubes were fitted to $\Delta E_{B}=E_{0}+A / R_{\mathrm{HC}}$ where $E_{0}$ and $A$ are $2.506 \mathrm{eV}$ and $-15.671 \mathrm{eV} \AA$, respectively. We note that for radius around $R_{\mathrm{HC}} \approx 6.25 \AA$, the $\Delta E_{B}$ becomes negative, suggesting instability. ${ }^{27}$ Hence, $(12,0)$ and $(8,8)$ nanotubes are at the limit for stable, fully exohydrogenated nanotubes. We are currently studying this problem for half-coverage case as well.

The energy, $\Delta E_{G}$, gained by attaching a single $\mathrm{H}$ atom to a carbon nanotube is calculated by performing structure optimization of a $\mathrm{C}_{n} \mathrm{H}$ nanotube as depicted in the bottom inset to Fig. 3(b). It is seen that $\Delta E_{G}$ can be also well described by
$\Delta E_{G}=E_{0}^{\prime}+A^{\prime} / R_{\mathrm{HC}}$ (dashed line) where $E_{0}^{\prime}$ and $A^{\prime}$ are $-1.161 \mathrm{eV}$ and $-4.9521 \mathrm{eV} \AA$, respectively. Unlike $\Delta E_{B}$, there is no change in the sign of $\Delta E_{G}$, suggesting that for any radius of carbon nanotube hybridization of a single carbon atom is always stable. However the energy gain from two such processes is around $5-6 \mathrm{eV}$, which is slightly less than the dissosiation energy of $\mathrm{H}_{2}, 6.65 \mathrm{eV}$. Hence the $\mathrm{C}_{n}$ nanotube plus $\mathrm{H}_{2}$ system is stable against forming a $\mathrm{C}_{n} \mathrm{H}_{2}$ hydrogenated nanotube. Therefore, in order to realize the $\mathrm{CH}$ bonding discussed here, one first has to break $\mathrm{H}_{2}$ molecules into hydrogen atoms, probably by using a metal catalyst or electrochemical techniques.

Hydrogenation of nanotubes is also important in the modification of the electronic structure for device applications. Figure 4 shows the band structure and the corresponding density of states (DOS) for a $(9,0)$ exohydrogenated nanotube, which is typical of other nanotubes that we studied. Using projected DOS, we find that the bottom of the conduction bands are mainly derived from hydrogen while the top of the valence bands are mainly of carbonorigin. In contrast to pure nanotubes, which are metal or semiconductors depending on their structure, the $\mathrm{C}_{n} \mathrm{H}_{n}$ nanotubes are found to be direct band insulators with a gap of $1.5-2 \mathrm{eV}$ at the $\Gamma$ point. This value is about one-third of those for the molecular polyhedrals, indicating less stability of hydrogenated nanotubes than molecules [Fig. 4(b)]. The band gaps decrease with increasing tube radius but unlike binding energies there is no apparent $1 / R_{\mathrm{HC}}$ type behavior. Interestingly, the band gaps of armchair nanotubes are higher in energy by about $0.2 \mathrm{eV}$ than those in zigzag nanotubes. This is surprising because the band gap is usually higher for more stable saturated hydrocarbons.

The observed band gap opening via hydrogenation of nanotubes can be used for band gap engineering for device applications such as metal-insulator heterojunctions. For example, various quantum structures can easily be realized on an individual carbon nanotube, and the properties of these structures can be controlled by partial hydrogenation of car- 

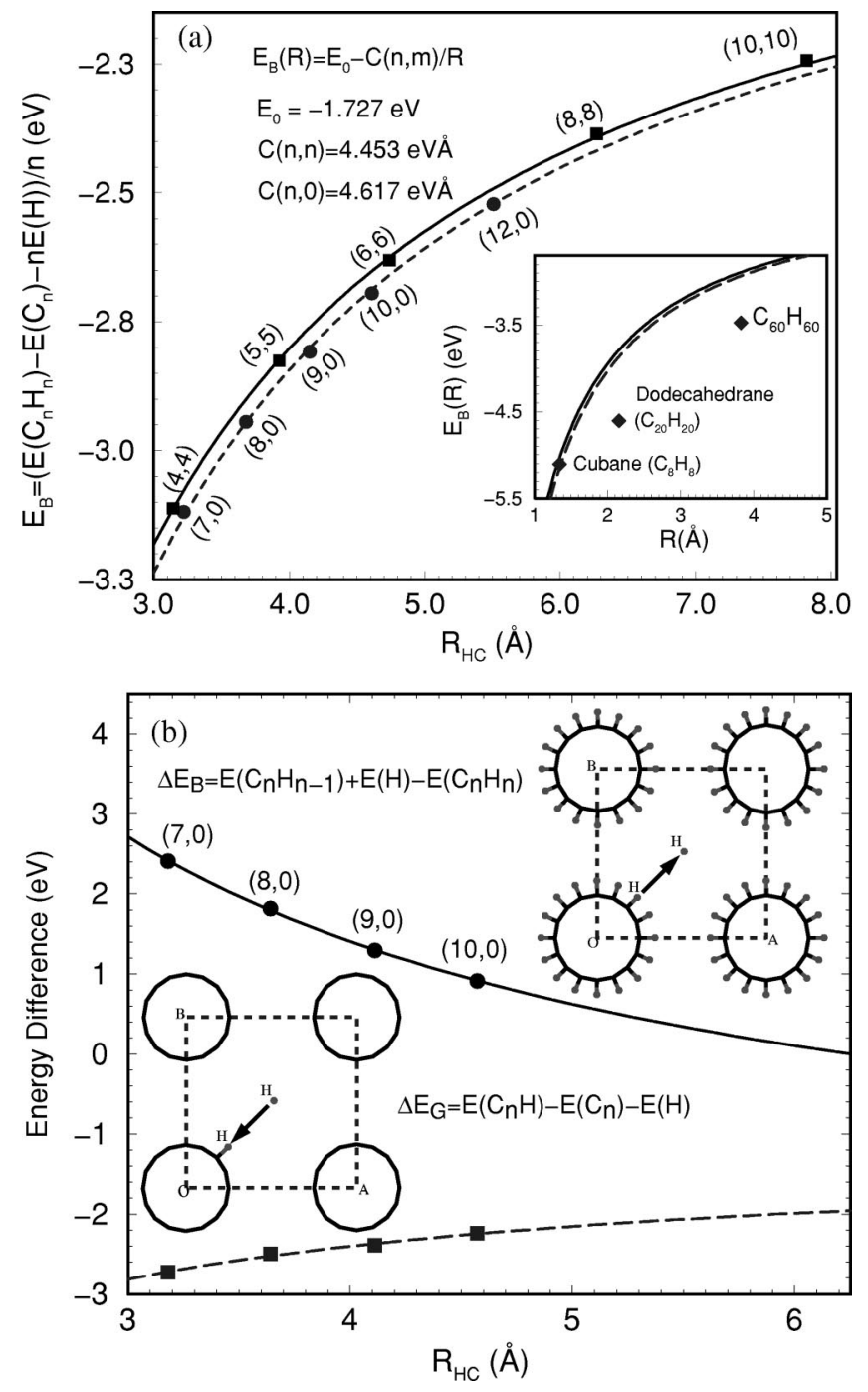

FIG. 3. (a) Binding energies $E_{B}$ of $(n, n)$ (square) and $(n, 0)$ (circle) nanotubes as a function of $R_{\mathrm{HC}}$. The solid and dashed lines are one-parameter fits to $E_{B}=E_{0}-C(n, m) / R_{\mathrm{HC}}$ as discussed in the text. Inset shows the binding energies of cubane, dodecahedrane, and $\mathrm{C}_{60} \mathrm{H}_{60}$. (b) Full circles indicate the energy $\left(\Delta E_{B}\right)$ to break a single $\mathrm{CH}$ bond to form a $\mathrm{C}_{n} \mathrm{H}_{n-1}$ zigzag nanotubes as depicted in the top inset. Full squares indicate energy gain $\Delta E_{G}$ by attaching a single $\mathrm{H}$ atom to a nanotube to form a $\mathrm{C}_{n} \mathrm{H}$ as depicted in the bottom inset. The solid and dashed lines are two-parameter fits as discussed in the text, indicating $1 / R_{\mathrm{HC}}$ behavior.

bon nanotubes. If the different regions of a single-wall nanotube are covered with hydrogen atoms, the band gap and hence the electronic structure will vary along the axis of the tube. This way various quantum structures of the desired size and electronic character can be formed. In this respect, present scheme is quite similar to our previous constructions of nanotube heterostructures or quantum dots, where periodic applied transverse compressive stress is used for the band gap opening. ${ }^{28}$

In summary, we have presented first-principles calculations of the structural and electronic properties of various nanotubes which are fully protonated by $s p^{3}$ hybridization of
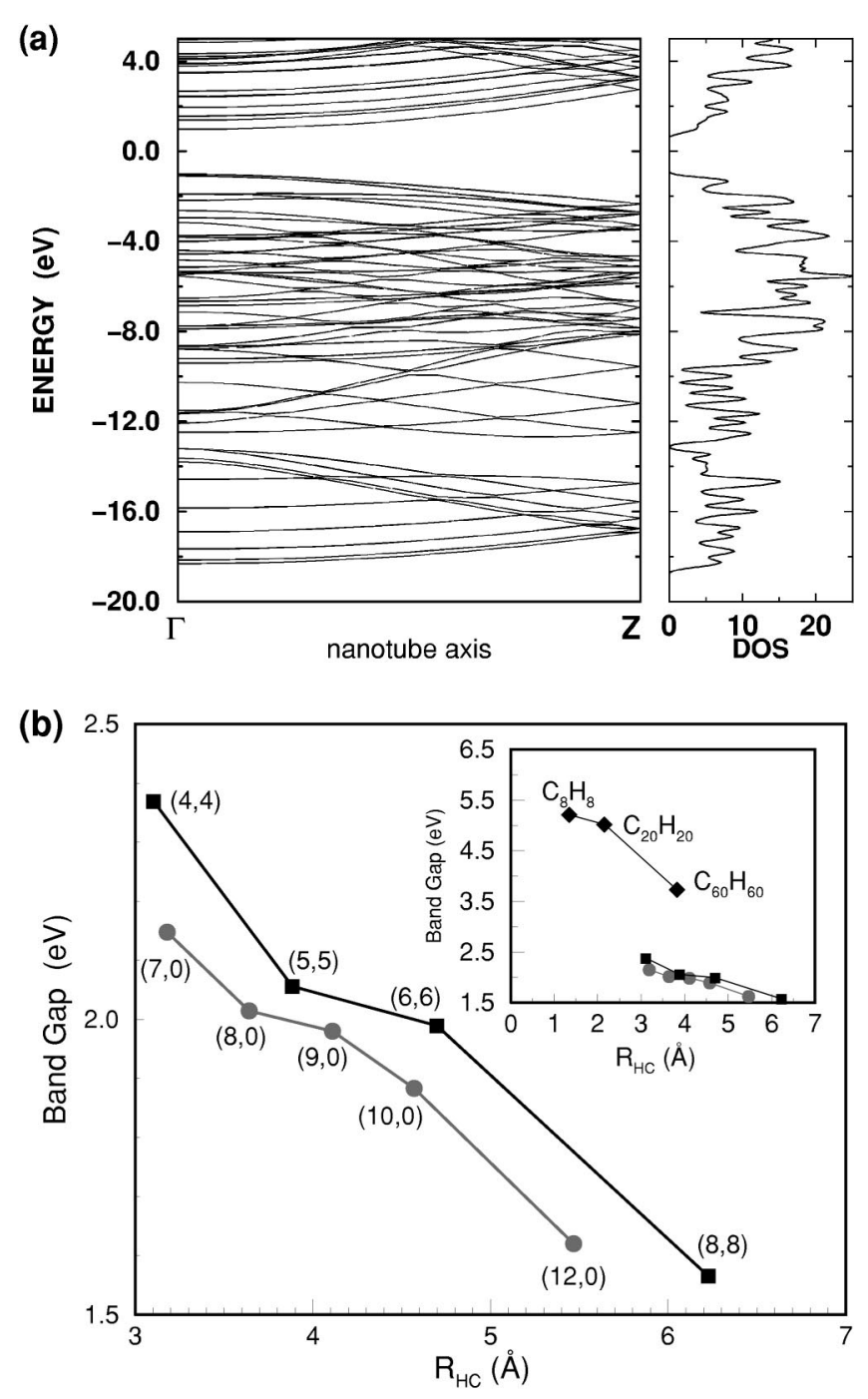

FIG. 4. (a) Electronic band structure of a $(9,0)$ exohydrogenated carbon nanotube (left panel) and the corresponding density of states (DOS) (right panel). (b) Band gap as a function of tube radius $R_{\mathrm{HC}}$. Inset shows the full scale plot to include the band gaps of the polyhedral molecules.

carbon atoms. We find that $\mathrm{C}_{n} \mathrm{H}_{n}$ nanotubes are stable for tube radius $R_{\mathrm{HC}}$ smaller than $6.25 \AA$, roughly corresponding to a $(8,8)$ nanotube. Hybridization of a single carbon atom is found to be always exothermic regardless of tube radius. Weak but stable $\mathrm{CH}$ bonding in nanotubes may be an important consideration for possible hydrogen storage applications. We also found that hybridization of zigzag nanotubes is more likely than armchair nanotubes with the same radius, suggesting a possible selective chemical functionalization of nanotubes. The fact that other carbon clusters such as cubane, dodecahedrane, and $\mathrm{C}_{60} \mathrm{H}_{32}$ have been synthesized successfully suggests that it may possible in the near future to hydrogenate carbon nanotubes, yielding new structures with novel properties.

This work was partially supported by the National Science Foundation under Grant No. INT97-31014 and TÜBITAK under Grant No. TBAG-1668 (197 T 116). 
${ }^{1}$ S. Iijima, Nature (London) 354, 56 (1991); S. Iijima, T. Ichihashi, and Y. Ando, ibid. 356, 776 (1992).

${ }^{2}$ N. Hamada, S. Sawada, and A. Oshiyama, Phys. Rev. Lett. 68, 1579 (1992).

${ }^{3}$ M.S. Dresselhaus, G. Dresselhaus, and P.C. Eklund, Science of Fullerenes and Carbon Nanotubes (Academic Press, San Diego, 1996).

${ }^{4}$ A.C. Dillon, K.M. Jones, T.A. Bekkadahl, C.H. Kiang, D.S. Bethune, and M.J. Heben, Nature (London) 386, 377 (1997).

${ }^{5}$ C. Liu, Y.Y. Fan, M. Liu, H.T. Cong, H.M. Cheng, and M.S. Dresselhaus, Science 286, 1127 (1999).

${ }^{6}$ C.M. Brown, T. Yildirim, D.A. Neumann, M.H. Heben, T. Gennett, A.C. Dillon, J.L. Alleman, and J.E. Fischer, Chem. Phys. Lett. 329, 311 (2000).

${ }^{7}$ C.C. Ahn, Y. Ye, B.V. Ratnakumar, C. Witham, R.C. Bowman, and B. Fultz, Appl. Phys. Lett. 73, 378 (1998).

${ }^{8}$ P. Chen, X. Wu, J. Lin, and K.L. Tan, Science 285, 91 (1999).

${ }^{9}$ Qinyu Wang, S.R. Challa, D.S. Sholl, and J.K. Johnson, Phys. Rev. Lett. 82, 956 (1999).

${ }^{10}$ R.C. Gordillo, J. Boronat, and J. Casulleras, Phys. Rev. Lett. 85, 2348 (2000).

${ }^{11}$ V.V. Simonyan, P. Diep, and J.K. Johnson, J. Chem. Phys. 111, 9778 (1999).

${ }^{12}$ K. Tada, S. Furuya, and K. Watanabe, Phys. Rev. B 63, 155405 (2001).

${ }^{13}$ Y. Ma, Y. Xia, M. Zhao, R. Wang, and L. Mei, Phys. Rev. B 63, 115422 (2001).

${ }^{14}$ P.E. Eaton and T.W. Cole, Jr., J. Am. Chem. Soc. 86, 962 (1964).
${ }^{15}$ T. Yildirim, P.M. Gehring, D.A. Neumann, P.E. Eaton, and T. Emrick, Phys. Rev. Lett. 78, 4938 (1997).

${ }^{16}$ T. Yildirim, S. Ciraci, and A. Buldum, Phys. Rev. B 62, 7625 (2000).

${ }^{17}$ B.S. Hudson, D.A. Braden, S.F. Parker, and H. Prinzbach, Angew. Chem. Int. Ed. Engl. 39, 514 (2000).

${ }^{18}$ M.I. Attalla, A.M. Vassallo, B.N. Tattam, and J.V. Hanna, J. Phys. Chem. 97, 6329 (1993).

${ }^{19}$ T. Guo and G.E. Scuseria, Chem. Phys. Lett. 191, 527 (1992).

${ }^{20}$ T. Yildirim, O. Gülseren, Ç. Kílíç, and S. Ciraci, Phys. Rev. B 62, 12648 (2000).

${ }^{21}$ Structure of a nanotube is described by two integers $(n, m)$ as explained in M.S. Dresselohaus, G. Dresselhaus, and R. Saito, Phys. Rev. B 45, 6234 (1992).

${ }^{22}$ M.C. Payne, M.P. Teter, D.C. Allen, T.A. Arias, and J.D. Joannopoulos, Rev. Mod. Phys. 64, 1045 (1992). The computer code CASTEP is distributed and maintained by Molecular Similations, Inc.

${ }^{23}$ J.P. Perdew and Y. Wang, Phys. Rev. B 46, 6671 (1992).

${ }^{24}$ D. Vanderbilt, Phys. Rev. B 41, 7892 (1990).

${ }^{25}$ H.J. Monkhorst and J.D. Pack, Phys. Rev. B 13, 5188 (1976).

${ }^{26}$ O. Gülseren, T. Yildirim, and S. Ciraci (unpublished).

${ }^{27}$ There is an energy barrier for breaking a single $\mathrm{CH}$-bond process from the fully hydrogenated carbon nanotube; $\Delta E_{B}$ is the energy difference between the initial and final states.

${ }^{28}$ Ç. Kílíç, S. Ciraci, O. Gülseren, and T. Yildirim, Phys. Rev. B 62, R16 345 (2000). 